\title{
Priming effects in perceptual classification
}

\author{
JOHN H. FLOWERS \\ University of Nebraska, Lincoln, Nebraska
}

\begin{abstract}
Priming stimuli that spatially flank a fixated target stimulus may cause either facilitation or interference with target classification, depending on experimental context. Two experiments demonstrated distinct effects of response compatibility and semantic congruity between flankers and target. Response competition occurred when targets were flanked by context stimuli associated with the opposite response, but this effect diminished when the target was delayed relative to the flankers. Facilitative priming by response-compatible flankers, in contrast, required prior exposure of the flankers, and was strongly influenced by the semantic congruity of flankers and targets. These differing time courses suggest that perceptual priming encompasses a variety of distinct underlying cognitive and motor events.
\end{abstract}

The ability to benefit from visual context that supports a perceptual interpretation of, and perhaps a motor response to, a visual stimulus is an extremely important characteristic of human and animal visual systems. Both in the natural environment of visually skilled animals and in human tasks such as reading, spatially and temporally adjacent visual events often contain correlated sources of information. The use of these correlations can potentially increase the efficiency of visual information processing considerably. However, since correlations among visual events in the real world are seldom perfect, the design of a visual system sensitive to surrounding context must deal with the potential costs of processing contextual information that is either irrelevant or misleading. Understanding the characteristics of selective attention that permit the use of beneficial context while minimizing both the overall processing load and interference from misleading context has been a continuing goal of experimental psychology that has taken on additional importance with recent efforts to design efficient artificial visual systems.

\section{Contextual Facilitation and \\ Inhibition of Perceptual Decisions}

When human observers are instructed to fixate a particular location in a visual display and make some type of classification response to a stimulus such as a character or word presented at that location, the reaction time (RT) for making a perceptual decision about the stimulus

This research was supported in part by National Science Foundation Grant IST-8319016 to John Flowers. I gratefully acknowledge the assistance of the following individuals in data collection and analysis: Kathy Fuson, Dave Janeczko, Mary Johanns, Doris Lohr, Dorie Reed. I also wish to thank Cal Garbin, Ken Paap, James Neely, James Pomerantz, and anonymous reviewers for helpful comments on an earlier version of this manuscript. Portions of this research were presented in a paper at the 56th Annual Meeting of the Midwestern Psychological Society, Chicago, IL, May, 1984. Requests for reprints should be sent to John H. Flowers, Department of Psychology, 209 Burnett Hall, University of Nebraska-Lincoln, Lincoln, NE 68588-0308. can be influenced by the presence of other stimuli in the display. This phenomenon is often called the flanker ef$f e c t$, since a typical stimulus display used to study it involves spatially flanking a letter stimulus with two or more noise or context letters. Even under conditions in which the noise or context stimuli that surround the target make no statistical prediction about the identity of the target, nor about the response that will most likely be required, substantial facilitative priming as well as substantial interference effects can be produced (B. A. Eriksen \& C. W. Eriksen, 1974; Flowers \& Wilcox, 1982; Grice, Boroughs, \& Canham, 1984; Taylor, 1977). Since these context effects occur even if the precise position of the stimulus target is prespecified and instructions are given to "ignore'" the context stimuli that flank the target, it is clear that human visual attention is not an ideally focused beam that excludes all processing of the context or nontarget stimuli. In addition, the fact that noise stimuli that flank a target can produce both Strooplike response competition effects and facilitation (when the noise elements are associated with same response as the target) indicates that noise stimuli receive sufficiently thorough or "deep" processing for response assignment information to be available.

A relatively straightforward and seemingly parsimonious interpretation of these flanker effects is that they are, like other forms of priming phenomena, the consequence of an involuntary (and obligatory) automatic activation (e.g., see LaBerge \& Samuels, 1974; Posner \& Snyder, 1975). This automatic activation by the flankers results in a direct response priming, which can produce facilitation when it primes the same response as the target, and inhibition when it primes a competing response. The greater prevalence of interference effects in flanker tasks (and other variants of the Stroop task) as opposed to lexical decision tasks (Neely, 1977) or sentence context tasks (Stanovich \& West, 1983) can be attributed to the fact that the flanker elements are directly associated with response alternatives rather than only providing activation of pathways necessary for stimulus interpretation; 
hence, response competition becomes a more salient component.

\section{The Time Course of Inhibition and Facilitation in Flanker Tasks}

Like both the Stroop variants and lexical decision priming experiments, the flanker tasks can involve asynchronous presentation of the priming or noise stimuli and the target stimulus. Through systematic manipulations of the relative onset time of the flankers and the noise (hereafter termed the stimulus onset asynchrony, or SOA), it is possible to show how facilitative and inhibitory effects of the flankers change over time, and thereby make inferences about the time course of stimulus coding and possible changes in the attentional state of the observer (C. W. Eriksen \& Shultz, 1979; Flowers \& Wilcox, 1982; Grice et al., 1984; Taylor, 1977).

With simultaneous onset of flankers and target, substantial response competition effects are typically noted in flanker tasks. For example, if subjects are required to press one response key if the target is an $\mathrm{H}$ or a $\mathrm{K}$, and another if it is an $\mathrm{S}$ or a $\mathrm{C}$, then response-incompatible target-flanker combinations (HSH) may produce RTs 30-50 msec slower than either response-compatible combinations (CSC) or various neutral character flanked or unflanked controls (B. A. Eriksen \& C. W. Eriksen, 1974; Grice \& Gwynne, 1985). In experiments in which SOA has been manipulated, the maximum amount of interference from response-incompatible flankers has typically occurred with either simultaneous onset or relatively short (e.g., < $100 \mathrm{msec}$ ) SOAs between flanker presentation and target onset, with a substantial drop in interference at longer SOAs. Several experiments have shown that response competition effects are virtually eliminated at SOAs longer than $300 \mathrm{msec}$ (Flowers, 1980; Flowers \& Wilcox, 1982; Grice et al., 1984; Taylor, 1977). Although the exact time relations vary with the type of stimulus material used, an attenuation of interference with increasing SOA is also typical of asychronous Stroop naming tasks, with the greatest interference noted for simultaneous or near-simultaneous onset of target and interfering attribute (M. O. Glaser \& W. R. Glaser, 1982; W. R. Glaser \& Dungelhoff, 1984).

Facilitation from flankers identical to (e.g., $\mathrm{HHH}$ ) or response-compatible with $(\mathrm{HKH})$ the target, on the other hand, follow a very different pattern as a function of SOA. Although some studies have resulted in a small amount of facilitation with simultaneous onset of targets and identical or response-compatible flankers (e.g., Taylor, 1977), most experiments have resulted in either RTs that did not differ significantly from RTs with a no-noise or neutral noise control (e.g., B. A. Eriksen \& C. W. Eriksen, 1974; C. W. Eriksen \& Hoffman, 1973) or even in a small interference effect (Flowers \& Wilcox, 1982; Grice et al., 1984; Grice \& Gwynne, 1985; Proctor \& Fober, 1985; Shaffer \& LaBerge, 1979). Some of the inconsistencies among the conclusions of these experiments may stem from different choices of the baseline condition against which small amounts of facilitation and interference can be evaluated. However, in two of the experiments, in which special care was taken to evaluate the consequences of different neutral or baseline conditions (Grice et al., 1984; Grice \& Gwynne, 1985), analyses of RT distributions as well as their means seem to have supported the existence of a small distractor effect as opposed to a facilitation effect when flankers are physically identical to the target.

Facilitation effects can be observed, however, when targets are flanked by identical letters, provided that the flankers lead the target in time. Although insufficient data exists to determine the minimum SOA necessary to produce facilitation (and such a value would probably vary with the type of stimuli used), previous data do suggest that facilitation from flankers identical to the target can occur when SOAs longer than 67-100 msec are employed (Flowers \& Wilcox, 1982). In addition, facilitation effects would appear to be maintained at somewhat longer SOAs (e.g., 200-300 msec) than those at which interference effects occur (Flowers \& Wilcox, 1982; Grice \& Gwynne, 1985). It should be noted that similar asymmetries in the functions relating SOA to facilitation and interference have been noted for the Stroop task as well (M. O. Glaser \& W. R. Glaser, 1982; W. R. Glaser \& Dungelhoff, 1984). Collectively, this evidence for different time courses for facilitation effects and interference suggests that these two varieties of context effects may involve somewhat different processing mechanisms, or at least an asymmetry in the behavior of the response mechanisms with respect to congruent as opposed to incongruent input.

\section{Semantic Congruity Effects}

Flanker effects are not exclusively limited to responsecompatibility effects, per se. Relatively few experiments have used stimuli selected from well-learned categories such as letters versus digits (Flowers \& Wilcox, 1982; Proctor \& Fober, 1985), or words belonging to different noun categories (Shaffer \& LaBerge, 1979). Although differences in procedure make it difficult to generalize across these studies, there is an indication that semantic category congruity between flankers and targets can influence stimulus classification time somewhat independently of response compatibility. For example, in a word classification task used by Shaffer \& LaBerge (1979), subjects were required to respond with one key if a target word belonged to the category of metals or clothing, and respond with another key to words from the category of furniture or trees. These target words were vertically flanked by words from the same or opposite response assignment. Physically different target-flanker combinations that were both response-compatible and semantically congruent (e.g., iron-zinc-iron) led to faster responding than response-compatible but categorically different combinations (iron-shirt-iron). Flowers and Wilcox (1982) and 
Proctor and Fober (1985) reported a similar semantic congruity effect with classification tasks requiring the sorting of mixtures of letters and digits into two response categories that varied orthogonally with the letter-digit distinction (e.g., D, N, 3, 7, vs. S, R, 4, 5).

If previously learned categorical information can contribute to flanker effects somewhat independently of response-compatibility effects, as these studies suggest, then "single process" interpretations of perceptual priming effects are inadequate. Furthermore, to the extent that these categorical influences act separately from responsecompatibility effects, there is interest in studying possible differences in the time course over which they arise. Of the studies mentioned above, the only flanker experiment in which SOA was manipulated with categorically heterogeneous stimuli (mixed letters and digits) was that of Flowers and Wilcox (1982, Experiment 3). In that experiment, facilitation produced by flanker-target combinations that were both response-compatible and categorically congruent (e.g., 454) produced faster responses than did combinations that were response-compatible, but not categorically congruent (e.g., S4S) - the latter type of stimulus displays produced response times about equal to those of neutral character flanked targets (e.g., ${ }^{*} 4 *$ ). A marginally significant interaction between SOA, response compatibility, and categorical congruity suggested that the influence of the letter-digit distinction was most pronounced at longer SOAs than at those which produced maximum response-compatibility effects per se, indicating that for this particular letter classification task, the availability of the categorical information (letters vs. digits) developed more slowly than that of response assignment information (left vs. right button). However, the marginal statistical reliability of the effect requires that replication, using a wider range of stimulus materials and SOA manipulations, should be undertaken before firm conclusions about differing time courses of responsecompatibility as opposed to categorical congruity effects can be made.

In summary, then, there exists scattered evidence from a variety of experiments that Strooplike responsecompetition effects and facilitative priming effects in perceptual classification tasks have different time courses of activation, and that facilitative priming may involve not only categorical congruity effects but also responsecompatibility effects. The present experiments were therefore conducted to examine how response-compatibility effects (both facilitative and inhibitory), as well as categorycongruity effects independent of response assignment, can jointly influence target classification time within a flanker task. A common task structure was used in these two experiments (mapping four target items into two categories, such that a "categorical" dimension varied orthogonally with the response assignments), but the choice of stimulus materials was varied. Experiment 1 required classification of letters and digits; words constituted the stimuli for Experiment 2.

\section{EXPERIMENT 1}

\section{Method}

Subjects. A total of 30 graduate and undergraduate students volunteered to participate in two experimental sessions, each lasting about $1 \mathrm{hr} 45 \mathrm{~min}$. These subjects were paid $\$ 14.00$ for participation. All subjects claimed to have normal or corrected-to-normal vision, and English was their native language.

Apparatus and Procedure. Experimental sessions were held in a dimly lit room, and subjects had about $5 \mathrm{~min}$ to adapt to the light before beginning the experiment. During this time, they were given experimental instructions (for Session 1), or the instructions were reviewed (Session 2). An Apple II + computer, modified to allow monitoring of the video synchronization signal through a game input port, controlled stimulus presentation and data acquisition. A Zenith model ZVM-121 video monitor (P31 phosphor), located on a desk at which the subject was seated, served as the stimulus display device. The distance between the subjects' eyes and the monitor screen was about $50 \mathrm{~cm}$, providing an angular separation between characters on the screen of about $.8^{\circ}$.

On each experimental trial, subjects observed the following sequence of events. First, a general warning signal, consisting of a display such as "GET READY FOR TRIAL \#1", was shown for a 3-sec period. Next, a fixation field consisting of a horizontal row of three plus signs $(+t+)$ was displayed in the location where the stimulus characters were to appear. This fixation field remained in view for approximately $2 \mathrm{sec}$, after which time the leftmost and rightmost $+s$ were replaced by flanking noise characters. The onset of this flanker field was synchronized with the video synchronization signal. After an SOA of $0,100,200,300$, or $500 \mathrm{msec}$ (all even multiples of the video scan rate), the target character replaced the center plus sign and remained in view until the subject responded by pressing a key on the computer console. A CCS 7440 programmable timer measured reaction time to the nearest millisecond, from the display onset until the keypress.

The target characters were selected from the set A, B, Y, Z, 1, $2,8,9$, while the noise characters were selected from the same set of digits and letters plus the neutral character " *'. Each of the target alternatives appeared equally often in combination with each noise character, so that there was no statistical correlation between any noise and target element. A typical display sequence on a given trial would have been:

$$
+++\rightarrow \mathrm{A}+\mathrm{A} \rightarrow \mathrm{A} 2 \mathrm{~A} \text {. }
$$

Trials were presented in blocks of 75 trials each, of which the first 3 trials in each block were excluded from analysis. During the entire experiment, each subject served in $\mathbf{3 0}$ such trial blocks, the first 5 of which were practice trials that were excluded from analysis. Each subject thus contributed a total of $25 \times 72=1,800$ experimental trials during the entire experiment.

The subjects were instructed to focus attention on the target and to ignore the characters appearing in the flanking positions. The subjects were also instructed to respond as rapidly as possible while avoiding errors. On trials in which the opposite key from that required by the classification rule was pressed, a blinking "ERROR!" message was displayed for about $3 \mathrm{sec}$ following the response. For responses shorter than $100 \mathrm{msec}$, the message "YOU JUMPED THE GUN" was shown for about $3 \mathrm{sec}$. On trials in which the response required more than $1,000 \mathrm{msec}$, the message "TOO LONG!" was shown. On any trial in which a subject accidentally struck a key other than the response keys ( $F$ or $J$ ), the computer beeped, and the flashing message "YOU HIT AN ILLEGAL KEY" was shown along with an instruction for the subject to check his or her hand position and then press the space bar to continue. 
Stimulus classification rules. The set of alternative target characters can be classified according to two conceptual or semantic classification schemes. The most salient of these is the letter-digit distinction. However, another (perhaps less natural and less overlearned) dimension is based on distance relationships within a list. A, B, 1, and 2 are elements at the beginnings of their respective learned sequences (the alphabet and digits), whereas $Y, Z, 8$, and 9 are at the end. It was my purpose to have at least one of these dimensions vary orthogonally with the stimulus classification rule applied by subjects, so that the influence of categorical structure on priming effects could be examined independently of responsecompatibility effects. Therefore, three different stimulus classification rules were assigned to three different groups of subjects. One group of 10 subjects was assigned to respond with the left hand (using the " $F$ "' key on the computer console) to the targets A, B, 1 , or 2 , and to respond with the right hand (using the " $J$ " key) to the targets $Y, Z, 8$, or 9 . For this classification rule, the letterdigit distinction varied orthogonally with the classification scheme, while the beginning-end dimension was perfectly correlated with the response mapping.

For the second group of 10 subjects, the response mapping consisted of A, B, 8, 9 versus $Y, Z, 1,2$. Here the letter-digit distinction varied orthogonally with the response assignment as in the first rule, whereas the beginning-end distinction was not correlated with response assignment. Since no categorical distinction was correlated with response assignment, this rule should be somewhat less easily coded into memory than the first rule, leading to overall slower responding. However, the categorical distinction between letters and digits within response categories might therefore be even more salient than for the first rule.

For the third group of 10 subjects, the response assignment was perfectly correlated with the letter-digit distinction (A, B, Y, Z vs. $1,2,8,9$ ). Thus the categorical dimension varying orthogonally with the response mapping for these subjects was the beginning-end distinction. Observation of a category congruity effect within response mappings for these subjects (e.g., if responses were faster to a display such as A BA than to A Y A) would show that flanker effects can result from the presence of a rather subtle aspect of conceptual organization even when the correlation of a much more salient conceptual dimension with the response mapping rule produces a highly S/R compatible task.

Design. In Experiment 1, three independent variables were manipulated. One, the classification rule, was a between-subjects variable. Three groups of 10 subjects each were assigned to the $\mathrm{AB} 12 / \mathrm{YZ} 89, \mathrm{AB} 89 / \mathrm{YZ} 12$, and ABYZ/1289 mappings, respectively. These classification rules were held constant throughout the experiment for each subject.

The second independent variable was SOA-the time delay between the onset of the flanking noise characters and the onset of the target. Five levels of SOA were used: 0, 100, 200,300, and $500 \mathrm{msec}$. SOA was a within-subjects variable, with all subjects receiving all five levels. However, SOA was held constant within trial blocks, and the subject was informed about the SOA level before beginning each block. The order of SOAs given to each subject was determined by a block randomization procedure, such that each SOA level was presented once before it was repeated.

The third independent variable was the flanker condition. For each classification rule, there are six logical groupings of conditions, based on the relationship between the target and the flanker. These are illustrated, along with exemplars, in Table 1 . In the identical condition (ID), the flanker and target were the same character. For the same-category/same-response condition (SCSR), the flanker was physically different from the target, but belonged to the same response mapping and the same semantic category as the target. For Classification Rules 1 and 2 , semantic category refers to the digit-letter distinction; for Classification Rule 3, it refers to distance relationships between the targets and flankers-i.e, the be-
Table 1

Examples of Stimulus Displays for Each Flanker Condition in Each Classification Rule Group in Experiment 1

\begin{tabular}{lccc}
\hline Condition & $\begin{array}{c}\text { Rule 1 } \\
\text { (AB12/YZ89) }\end{array}$ & $\begin{array}{c}\text { Rule 1 } \\
\text { (AB89/YZ12) }\end{array}$ & $\begin{array}{c}\text { Rule 3 } \\
\text { (ABYZ/1289) }\end{array}$ \\
\hline ID & AAA & AAA & AAA \\
SCSR & BAB & BAB & BAB \\
N & ${ }^{*} A^{*}$ & ${ }^{*} A^{*}$ & ${ }^{*} A^{*}$ \\
SCDR & YAY & YAY & 1 A1 \\
DCSR & $1 A 1$ & $8 A 8$ & YAY \\
DCDR & $8 A 8$ & $1 A 1$ & $8 A 8$ \\
\hline
\end{tabular}

ginning-end distinction. The third condition was the neutral or baseline condition $(\mathrm{N})$. This condition represented the trials on which the target was flanked by asterisks; it served as a reference condition to evaluate facilitation or interference effects in other conditions. The fourth flanker condition was the same-category/differentresponse condition (SCDR). In this condition, the flanker and target were from the same semantic category (both of them digits or both of them letters for Classification Rules 1 and 2, and both of them beginnings or both of them ends for Classification Rule 3. The fifth flanker condition was the different-category/same-response condition (DCSR). On these trials, the target and flanker belonged to the same response assignment but belonged to different semantic categories. For the final flanker condition, differentcategory/different-response (DCDR), both the response assignments and semantic categories of the flanker and target differed. ${ }^{1}$

Since each flanker character was paired equally often with each target character (once within each 72 experimental trials within each block), so as to produce no statistical prediction of the target on the basis of the flanker, the ID and SCSR condition occurred half as often as the remaining flanker conditions ( 8 as opposed to 16 times per trial block, or 40 as opposed to 80 times per SOA level per subject). The order of conditions within trial blocks was determined by a computer randomization program that also chose 3 trials from among the 72-trial sequence, to serve as the 3 practice or warmup trials at the beginning of a block.

\section{Results and Discussion}

Figures 1-3 present, for the three classification rule groups, respectively, the mean RT for each flanker condition at each SOA. For clarity, each graph has been separated into two panels. The top panel of each graph provides a comparison between each of the responseincompatible flanker conditions (SCDR and DCDR) and the neutral condition $(\mathrm{N})$, while the bottom panel displays Condition $\mathrm{N}$ along with the response-compatible flanker conditions (ID, SCSR, and SCSR). Figure 4 displays the differences in RTs between the neutral $(\mathrm{N})$ condition and the other flanker conditions as a function of SOA, using data from all three classification rules combined. This graph thus provides a summary of interference or facilitation caused by the flankers relative to the presumably neutral asterisk.

Overall flanker effects. It is apparent from Figures 1-3 that substantial flanker effects occurred for each of the classification rule groups, and that although there were some minor differences between groups in the magnitude of these effects or in their interactions with SOA, there are several important characteristics of these data common among all three groups. These include generally 

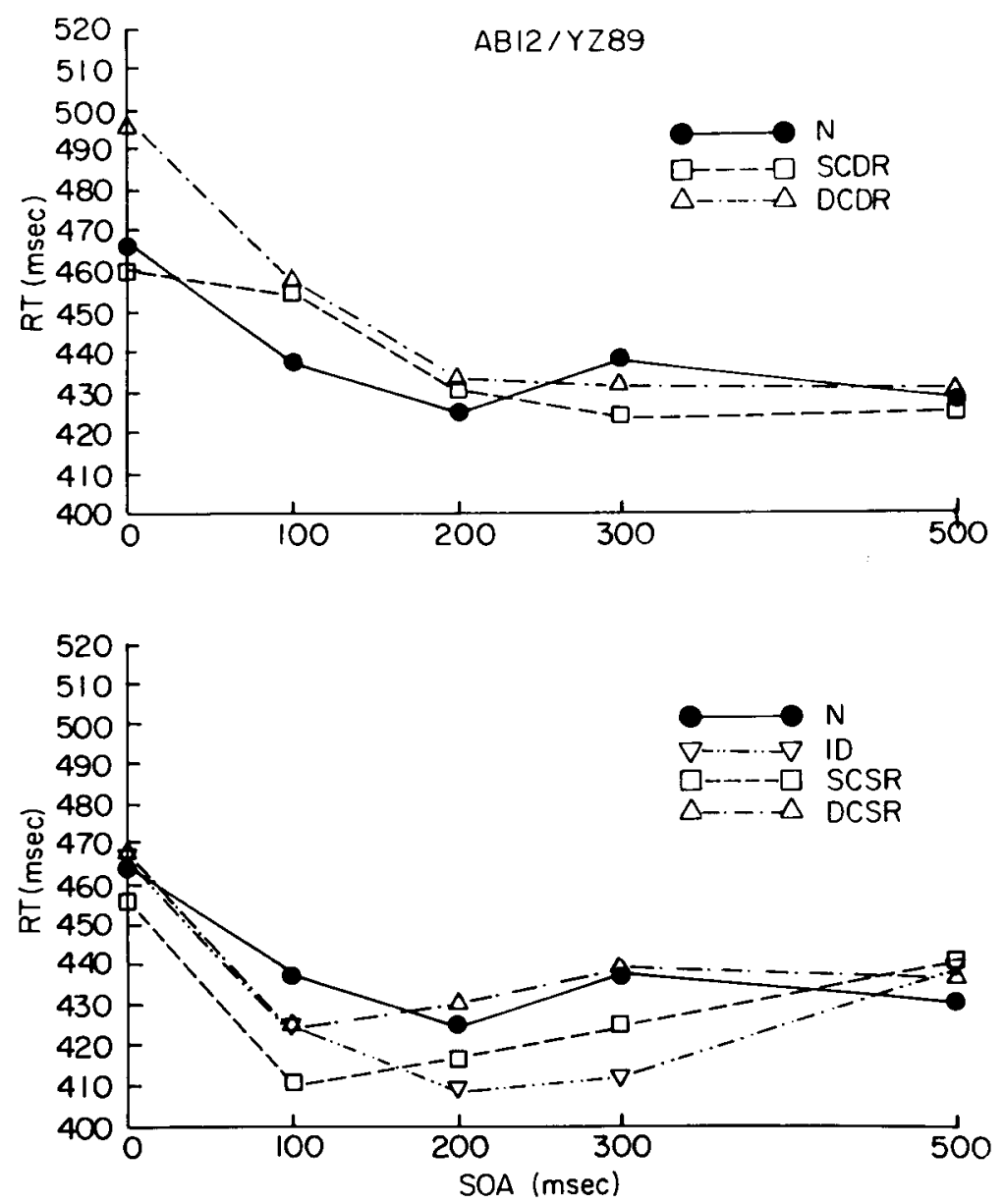

Figure 1. Mean reaction times as a function of flanker condition and SOA for the AB12/YZ89 classification task of Experiment 1. Top panel compares responseincompatible conditions with the neutral flanker condition, while the bottom panel compares the response-compatible conditions with the neutral condition.

shorter RTs for positive SOA conditions than for 0 SOA, which demonstrates that the flankers have a general alerting effect, irrespective of their form and relation to the target. Similar alerting effects have been noted in other experiments (e.g., Grice et al., 1984). Other specific flanker effects include both interference from response competition and facilitation from flankers that are both response-compatible and semantically congruent with the target. An overall three-way ANOVA (flanker type $x$ SOA $\times$ group, with subjects nested with groups) illustrated a highly significant overall effect of flanker type $[F(5,135)=14.6, p<.001]$, and a flanker type $\times$ SOA interaction $[F(20,540)=11.68, p<.001]$. Because of the existence of a marginally significant group $\times$ flanker type interaction $[F(10,135)=1.99, p<.05]$, as well as a group $\times$ flanker type $\times$ SOA interaction $[F(40,540)=2.87, p<.001]$, I also performed ANOVAs on each classification rule group separately, and significant effects of flanker type and SOA, as well as the significant interaction, existed for each group separately. ${ }^{2}$ Though there are thus some differences in the precise pattern of flanker effects across SOA that are seemingly attributable to individual classification rules, the general conclusion that specific flanker effects have different time courses seems to be warranted in each case. Also, there are some common features of the flanker effects among the three classification rules groups-namely, general characteristics of the time course of interference and facilitation effects, as well as indication of a distinction between effect of response compatibility and semantic congruity-that merit additional discussion.

Time course of interference and facilitation. Comparison of the upper and lower panels of Figure 4 will illustrate the differing influence of SOA on interference effects caused by response-incompatible flankers and facilitative priming resulting from response-compatible (and also semantically congruent) flankers. Interference effects were largest with simultaneous presentation of flankers and targets and essentially vanished when the flankers led the target by $200 \mathrm{msec}$. Facilitation effects, on the other hand, were largely absent at 0 -msec SOA and reached their maximum values at $200-300 \mathrm{msec}$. 
$\mathrm{AB89} / \mathrm{YZI2}$
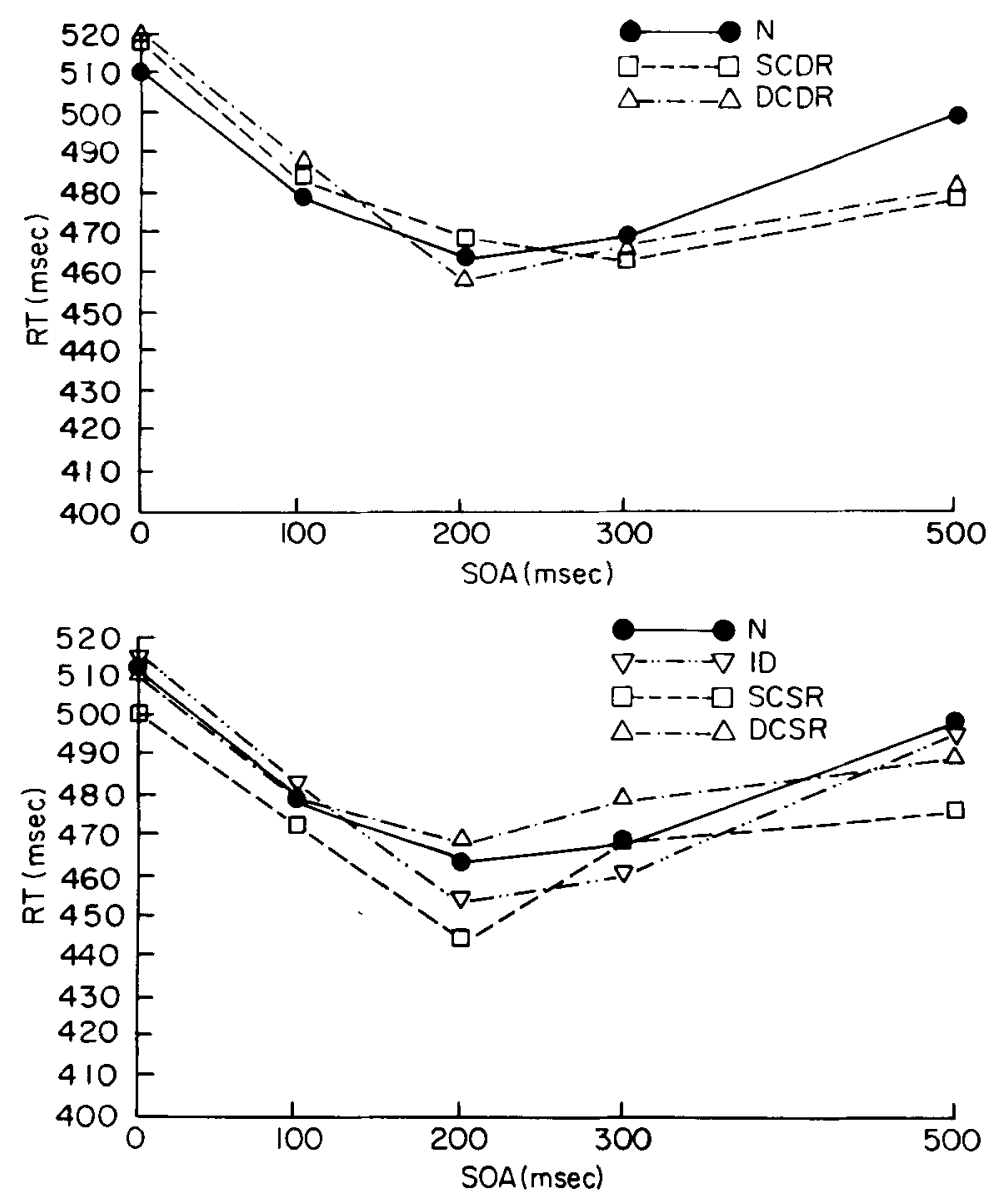

Figure 2. Mean reaction times as a function of flanker condition and SOA for the AB89/YZ12 classification rule of Experiment 1.

With SOAs as long as $500 \mathrm{msec}$, all forms of flanker effects are diminished. These findings are consistent with findings of Flowers and Wilcox (1982), Grice et al. (1984), and Taylor (1977), and add support for the conclusion that interference effects and facilitative priming may represent different phenomena.

Response compatibility and semantic congruity. Of particular interest in this experiment are the relative contributions of response compatibility and semantic congruity to the flanker effects, and whether these two factors are differentially affected by SOA. In order to address these issues, I conducted a preplanned ANOVA using a subset of the flanker conditions, which included SCSR, DCSR, SCDR, and DCDR. These four conditions were treated as a $2 \times 2 \times 5 \times 3$ factorial combination of response compatibility, semantic congruity, and SOA, with classification rule as a between-groups factor. Highly significant main effects of response compatibility $[F(1,27)=23.69, p<.001]$ and semantic congruity $[F(1,27)=89.27, p<.01]$ were found, and neither of the factors produced a significant interaction with classification rule group. ${ }^{3}$ The issues of particular interest in this comparison, however, are (1) whether the influence of semantic congruity is, as Flowers and Wilcox (1982) had found, dependent on the presence of response compatibility, and (2) whether semantic congruity might have a different time course of activation than the responsecompatibility effects have. A significant interaction between semantic congruity and response compatibility was found $[F(1,27)=5.01, p<.05]$, as well as a three-way interaction between these factors and SOA $[F(4,108)=$ $7.08, p<.01]$. Neither of these effects significantly interacted with classification rule group. Inspection of Figure 4 (as well as the mean reaction times in Figures 1-3) supports the general pattern observed by Flowers and Wilcox (1982, Experiment 3), that semantic congruity effects were largely limited to flanker-target combinations that also were response-compatible. As in the Flowers and Wilcox experiment, one of the most robust differences among flanker conditions was the difference between DCSR and the two conditions that were both responsecompatible and semantically congruent, ID and SCSR. It should also be noted that in both the Flowers and Wilcox experiment and the present case, this difference is 

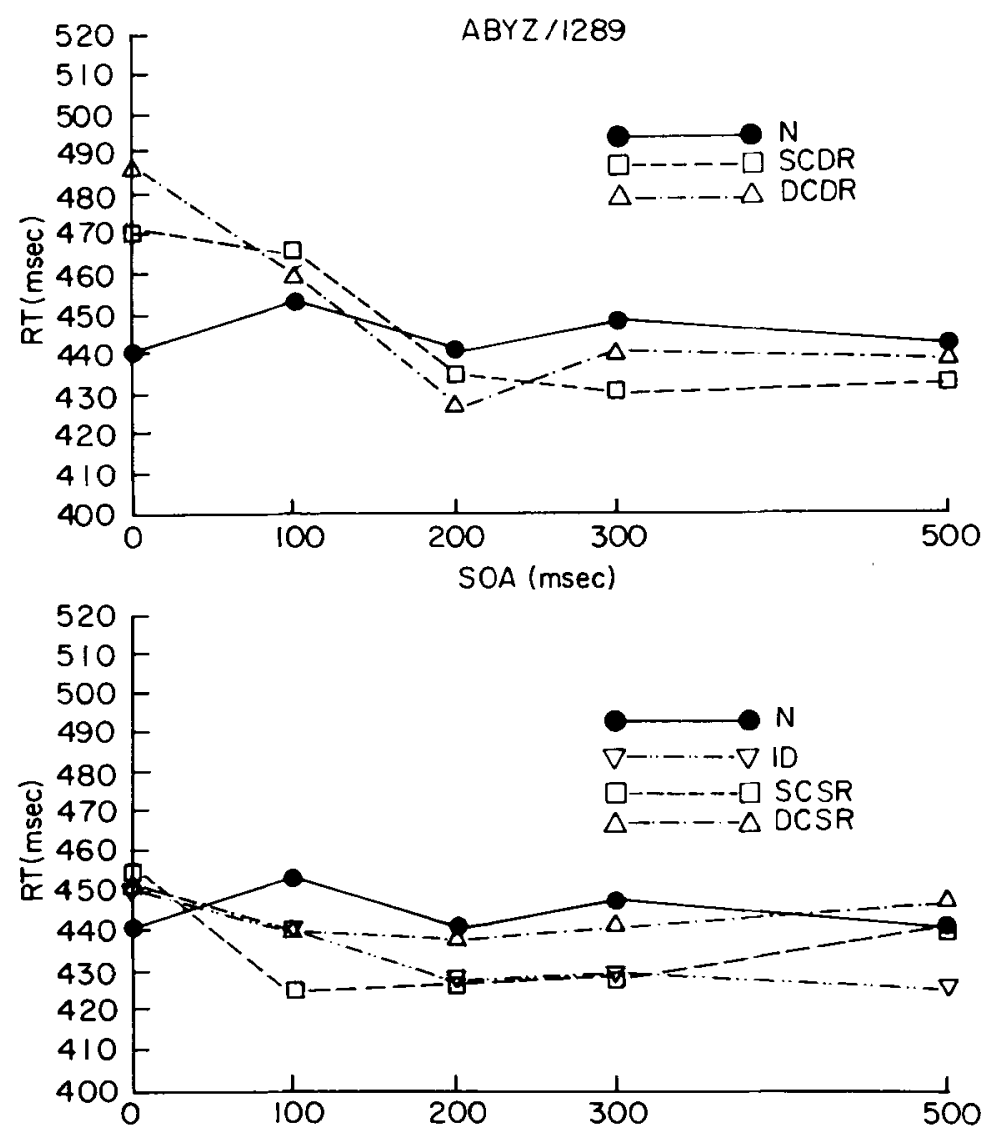

Figure 3. Mean reaction times as a function of flanker condition and SOA for the ABYZ/1289 classification rule of Experiment 1.

limited to the longer SOA levels, suggesting the possibility that semantic category information does not become available to the response process until more than $100 \mathrm{msec}$ after presentation of the flankers. This is in sharp contrast to the response competition effects that are most robust at $0 \mathrm{SOA}$, and have largely vanished by $200-\mathrm{msec}$ SOA.

At 0 SOA only, condition DCDR produced longer reaction times than did SCDR, and the magnitude of this difference (for Rules 1 and 3 ) is sufficiently large to suggest a possible real effect. On the other hand, this effect was negligible for Classification Rule 2, as well as in the data from Experiment 3 of Flowers and Wilcox (1982). Given these inconsistencies, evidence that semantic congruity affects the magnitude of interference effects in these types of tasks is tenuous-although "semantic distance" effects on Stroop interference have been previously reported (Flowers, Nelson, Carson, \& Larsen, 1984; Hock \& Petrasek, 1973). It does seem apparent, however, from the significant interaction between response compatibility, semantic congruity, and SOA, that whatever effects semantic congruity might have in the processing of displays that are response-incompatible, the time course of such effects is much different from that observed with response-compatible displays.
ID-SCSR differences. The present data illustrate a trend found by Flowers and Wilcox (1982), LaBerge (1981, p. 64), and Proctor and Fober (1985), that displays in which flankers are identical to the target lead to slower RTs at 0 and short SOAs than do displays in which flankers are physically different but both responsecompatible and semantically congruent with the target. A comparison of Conditions ID and SCSR across SOA illustrated a significant interaction with SOA $[F(4,108)=$ $4.25, p<.005$ ], showing that the effect of longer RTs for Condition ID at the shortest SOA levels, but not thereafter, was reliable.

Recently, Proctor and Fober (1985) have shown that this "repeated stimulus inferiority effect" may be unique to tasks such as the present one, in which there are mixtures of letters and digits within response categories. Nonidentical but response-compatible flankers typically produce slower reaction times than identical flankers when the target stimuli are all letters or all digits. Interpretation of this somewhat counterintuitive finding is problematic. I suggested (in Flowers \& Wilcox, 1982) that at 0 or very short SOAs, some form of perceptual feature competition effect might cancel out potential benefits of facilitative priming from response compatibility or semantic category congruity. Because the SCSR con- 

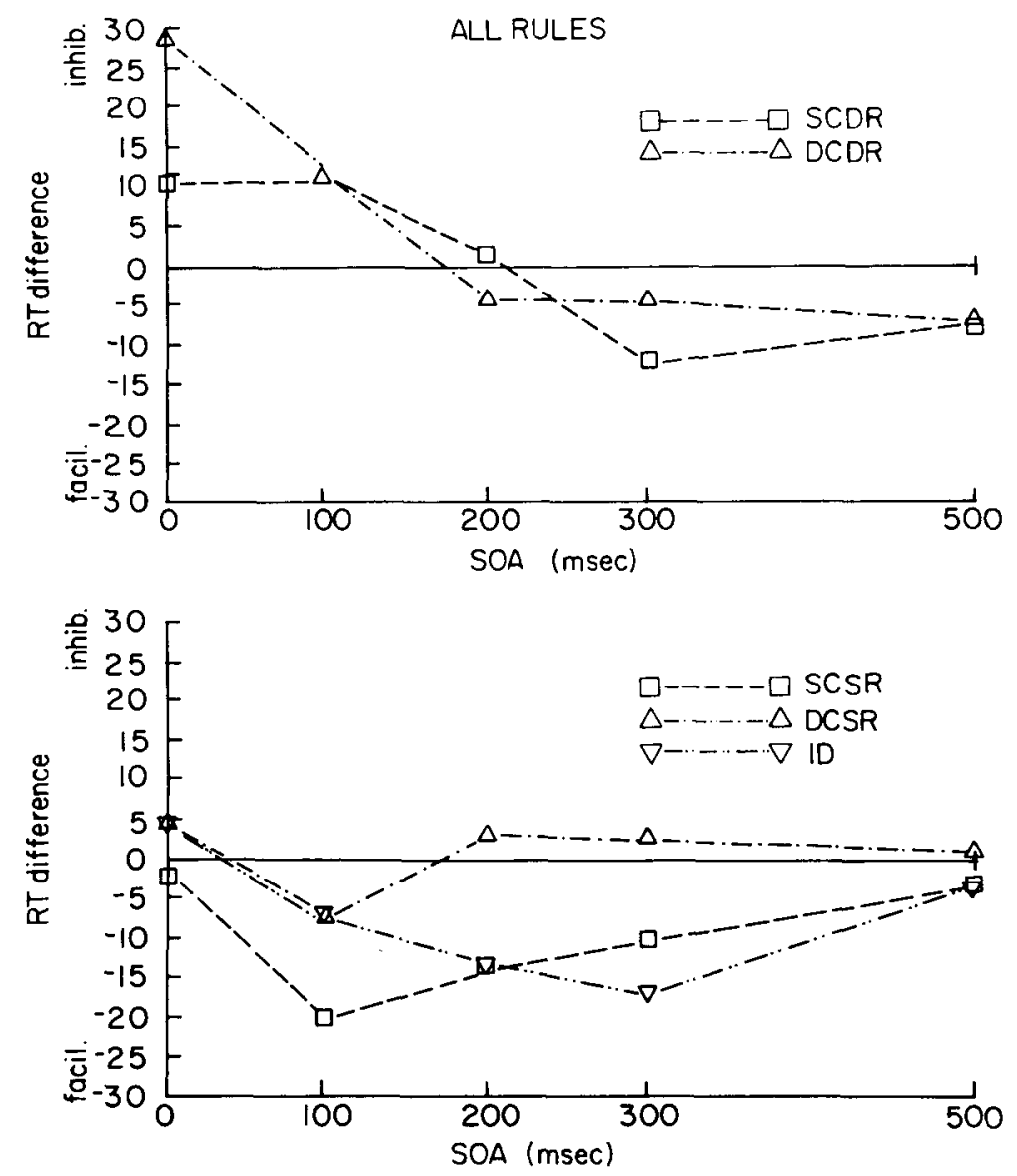

Figure 4. Differences in reaction time between the neutral and each of the other flanker conditions in Experiment 1, as a function of SOA. Differences are averaged across all three classification rules.

dition involves physically different flankers and targets, but still benefits from response compatibility and semantic congruity, faster RTs result. On the other hand, failure to find any additional evidence for feature competition based on slowing of reaction time in unmixed lists when flankers and targets are identical (Grice \& Gwynne, 1985; Proctor \& Fober, 1985) casts serious doubt on that interpretation, and thus I currently agree with Proctor and Fober that the basis for this repeated stimulus inferiority effect, which seems to be unique to tasks involving mixtures of digits and letters, is unclear.

Error rates. As is typical with tasks of this type, error rates were low and correlated with response latency. They ranged from under $3 \%$ for the response-compatible conditions at middle SOA levels to about $6.0 \%$ for the DCDR condition at short SOAs. Several subjects exhibited errorless performance in several conditions. Since errors were infrequent and provided no information beyond that of latency data, they were not subjected to formal analysis.

\section{EXPERIMENT 2}

In place of the letter and digit character stimuli used in Experiment 1, Experiment 2 required subjects to clas- sify common English nouns, selected to have a wellknown category structure that was mapped into response categories in a manner similar to the response mappings of Experiment 1. Flanker effects have been shown to occur with word stimuli by Shaffer and LaBerge (1979), and these effects included semantic congruity effects as well as response mapping effects. However, the Shaffer and LaBerge study was restricted to simultaneous presentation of target and flanker words, so that potential differences in the time courses of semantic and response priming could not be assessed.

\section{Method}

Subjects. Twenty-four undergraduate and graduate students each served in two experimental sessions lasting about $1 \mathrm{~h} 15 \mathrm{~min}$ each. These subjects were paid $\$ 9.00$ for their participation. All subjects claimed to have normal or corrected-to-normal vision and English as their native language.

Procedure and design. The general procedure and equipment used in Experiment 2 were identical to those of Experiment 1, with the exception of the stimulus materials, the classification rules, and the SOA levels chosen. Instead of classifying a character target that was horizontally flanked by noise characters, subjects classified uppercase word stimuli that were vertically flanked by noise words. On each experimental trial, the subjects viewed the following set 
of events: First, a "GET READY" message was shown. Then, a fixation field consisting of three vertically stacked rows of dashes was shown for approximately $2 \mathrm{sec}$. At this point, the top and bottom rows of dashes were replaced by the flanker word. Following the SOA, the target word replaced the middle row of dashes. At that point, the subject responded by pressing the " $F$ " or "'J' key to indicate classification of the target word into its response category. Figure 5 illustrates a sample stimulus display sequence. Horizontal spacing of the letters in the words was about $.8^{\circ}$, while the vertical visual angle between the rows was about $1.1^{\circ}$.

Two groups of 12 subjects were respectively assigned to two different word classification tasks. The first classification task mapped the words SILVER, GOLD, TABLE, and CHAIR to the left-hand response ("F"' key), and the words IRON, BRASS, LAMP, and PHONE to the right-hand response ("J" key). Thus, for this classification rule, the semantic dimensions of metals and furniture varied orthogonally with the response assignment. For the other classification rule, the words MAPLE, BIRCH, HORSE, and COW were mapped to the left-hand response, whereas SPRUCE, PINE, SHEEP, and GOAT were mapped to the righthand response. For this classification rule, the semantic categories of trees and animals varied orthogonally with the response assignment. ${ }^{4}$ The flanker words used were the same eight words as the target alternatives plus the letter string $\mathrm{XXXXX}$, which served as the neutral or baseline condition. Each target was paired equally often with each flanker alternative (once per each 72-trial block). Logical groupings of target-flanker combinations analogous to those of Experiment 1 were used to evaluate the different types of potential priming effects. Exemplars of these flanker conditions for each of the two classification tasks are shown in Table 2 . Since subjects produced data from five different trial blocks at each SOA level, the mean reaction time for each subject at each SOA level was based on a total of 40 trials for Conditions ID and SCSR, and 80 trials for the remaining flanker conditions.

The four SOA levels (lead time of the flankers) used in Experiment 2 were $0,100,250$, and 500 msec. These were held constant during trial blocks, and each SOA level was presented once (order determined by a Latin square) before it was repeated.

Feedback about errors was identical to that in Experiment 1, with the exception that response latencies of up to $1,200 \mathrm{msec}$ were tolerated before such trials were flagged as "too long." Stray keypresses, "too long" errors, and "gun-jumping" errors constituted less than $1.0 \%$ of all trials. As in Experiment 1, several subjects exhibited errorless performance for a majority of the flanker conditions at all SOAs. Misclassification errors varied from $1.9 \%$ (for SCSR at 100 -msec SOA) to $8.1 \%$ (for SCDR at 100 -msec SOA) across flanker conditions. Because of this low rate of occurrence, errors were not subjected to formal analysis. Only data from correct trials were used in computing mean RTs for each subject.

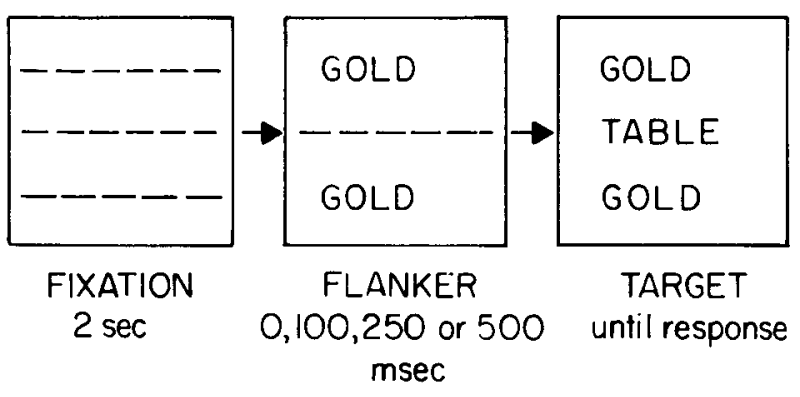

Figure 5. A sample display sequence from Experiment 2.
Table 2

Examples of Stimulus Displays for Each Flanker Condition in Each Classification Group in Experiment 2

\begin{tabular}{ccc}
\hline Condition & $\begin{array}{c}\text { Rule 1 } \\
\text { (Metals \& Furniture)* }\end{array}$ & $\begin{array}{c}\text { Rule 2 } \\
\text { (Trees \& Animals) } \dagger\end{array}$ \\
\hline ID & SILVER & MAPLE \\
& SILVER & MAPLE \\
SCSR & SILVER & MAPLE \\
& GOLD & BIRCH \\
& SILVER & MAPLE \\
N & GOLD & BIRCH \\
& XXXXX & XXXXX \\
& SILVER & BIRCH \\
SCDR & XXXXX & XXXXX \\
& SILVER & SPRUCE \\
& IRON & BIRCH \\
DCSR & SILVER & SPRUCE \\
& GOLD & HORSE \\
& TABLE & MAPLE \\
& GOLD & HORSE \\
DCDR & IRON & HORSE \\
& TABLE & SPRUCE \\
& IRON & HORSE
\end{tabular}

* Classification rule: SILVER, GOLD, TABLE, CHAIR/IRON, BRASS, LAMP, PHONE. + Classification rule: MAPLE, BIRCH, HORSE, COW/SPRUCE, PINE, SHEEP, GOAT.

\section{Results and Discussion}

Figures 6 and 7 display the mean RTs as a function of flanker condition across SOA for the two classification tasks. As in Experiment 1, the data from responsecompatible and response-incompatible trials are plotted together with the $\mathrm{N}$ condition in separate panels for clarity. In addition, Figure 8 shows the differences in mean RT between each experimental flanker condition and the $\mathrm{N}$ condition (XXXXX flanker trials) as a function of SOA, averaged across both classification tasks. This figure can (with appropriate caution about assuming the "neutrality" of the $\mathrm{N}$ condition, as discussed below) be used to evaluate the time course of facilitative priming and interference effects.

An ANOVA of the mean RTs showed significant main effects for flanker conditions $[F(5,110)=4.82, p<$ $.001]$, SOA $[F(3,66)=27.86, p<.001]$, and the SOA $\times$ flanker condition interaction $[F(15,330)=2.77, p<$ $.01]$. Although a visual comparison of Figures 6 and 7 suggests some minor differences in the pattern obtained with the two classification tasks, the ANOVA using the combined data from both groups showed that the classification rule variable was not involved in any significant interaction with any other variable $(F<1.45$ for all comparisons). Thus it may be assumed that differences among flanker conditions or other variables reflect a relatively similar structure among both classification tasks. With some exceptions to be noted below, this structure bears a great deal of similarity to the pattern obtained in Experiment 1 . 

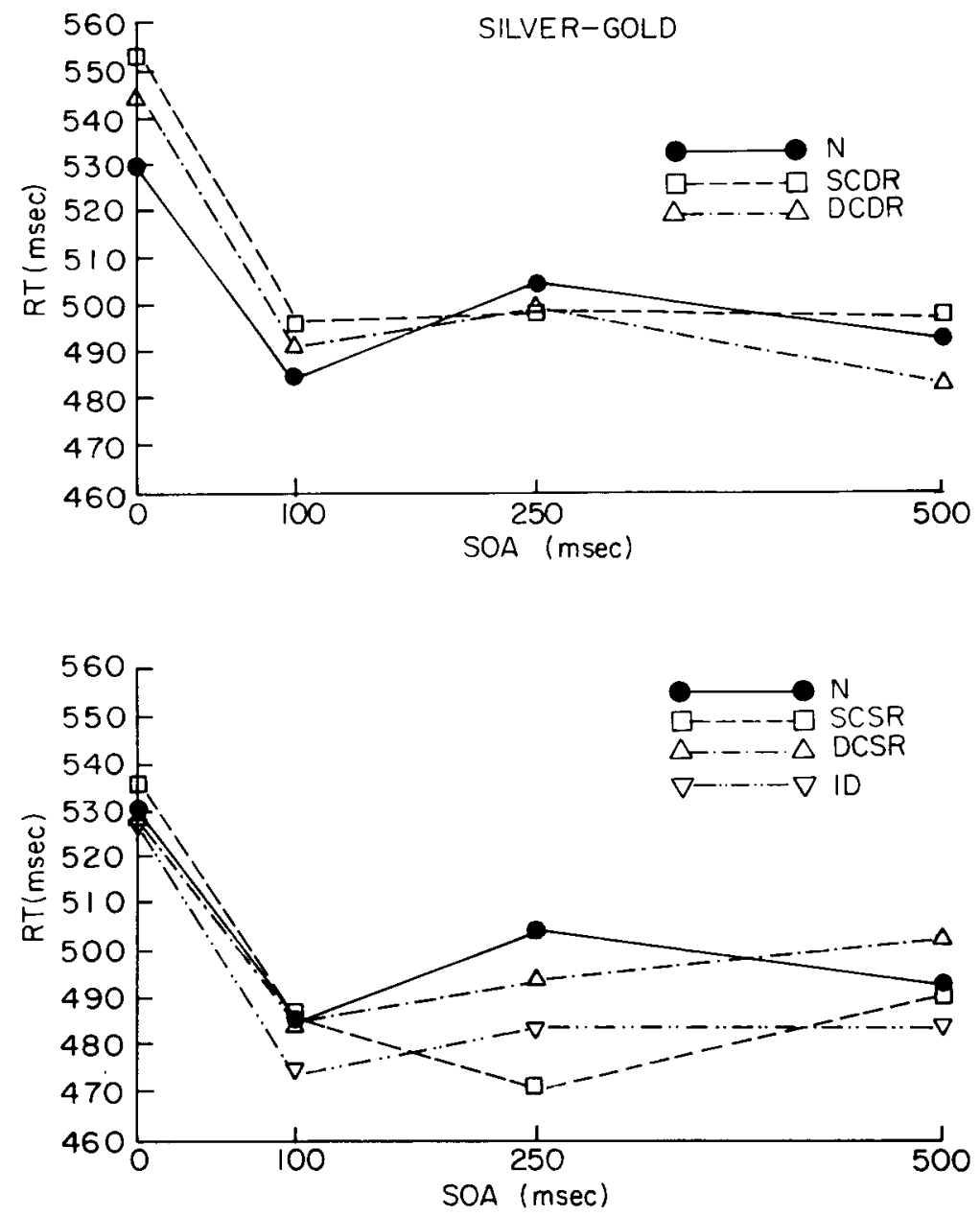

Figure 6. Mean reaction times as a function of flanker condition and SOA for the metals and furniture classification rule of Experiment 2.

One difference between the present results and those of Experiment 1 consists of the diminished interference effects attributable to response competition, as assessed by comparing conditions SCDR and DCDR to the N condition. In fact, comparisons between SCDR and $\mathrm{N}$ and between DCDR and $\mathrm{N}$ do not reach statistical significance at any of the SOA levels (a difference of $13.0 \mathrm{msec}$ would be required for a one-tailed test at a .05 alpha level, using the standard error computed from group data with 7.35 and $22 d f$ ). This lack of significance may be attributable, in part, to perceptual distraction caused by the XXXX flanker, which made it less "neutral" than the asterisk symbols used in the first experiment-as was suggested by subjective comments from several subjects.

There is, however, evidence that response compatibility did contribute significantly to the overall flanker effects, as evidenced by a comparison of responsecompatible with response-incompatible trials, exclusive of the neutral condition. As in Experiment 1, I conducted a planned $2 \times 2$ comparison of Conditions SCSR, SCDR, DCSR, and DCDR across SOA using the data from both classification rule groups. This comparison showed both a significant effect of response compatibility $[F(1,22)=$ $4.47, p<.05]$ and an interaction between SOA and response compatibility $[F(3.66)=6.10, p<.001]$. This interaction shows that, as in Experiment 1, responsecompatibility effects were most pronounced at the shorter SOAs.

Category-compatibility effects were evident in this comparison, but only at the longer SOAs, and they were limited to the flanker conditions that were also responsecompatible. Although overall, the $2 \times 2$ comparison showed no significant effect of category congruity, there was a significant interaction between response compatibility and category congruity $[F(1,22)=11.31, p<.01]$, an interaction between SOA and category congruity $[F(3.66)=2.46, p<.05]$, and a three-way interaction between SOA, response compatibility, and category congruity $[F(1,22)=11.31, p<.05]$. The most striking feature of the functions relating each flanker condition to SOA is the departure of condition DCSR from the other response-compatible conditions at the longer SOAs. This 

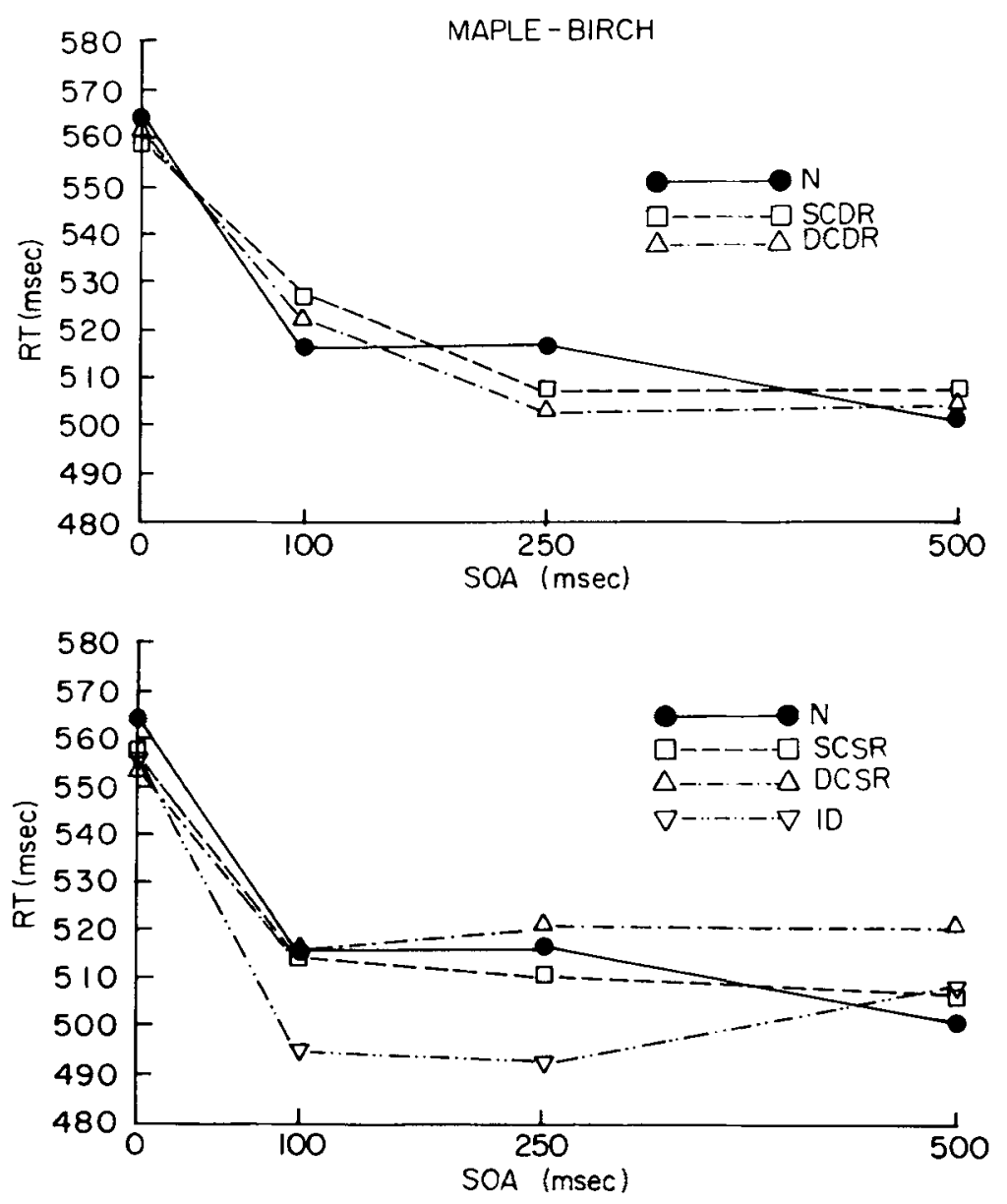

Figure 7. Mean reaction times as a function of flanker condition and SOA for the animals and trees classification rule of Experiment 2.

result is very similar to that obtained in Experiment 1, as well as in Experiment 3 of Flowers and Wilcox (1982); it shows that the categorical congruity between a flanker and target can strongly influence RT-most potently at longer SOAs than those which are sensitive to responsecompatibility effects. A second minor discrepancy between the character classification tasks and the word classification tasks of the present experiment concerns differences between the ID and SCSR conditions. In Experiment 1 , facilitation with the ID conditions developed more slowly across SOA than did facilitation by the SCSR conditions (suggesting a possible repeated signals "inferiority effect" at short SOAs), whereas such was clearly not the case in Experiment 2. It is possible that the relationship between these two conditions across SOA may be related to the speed with which the semantic categorization can be made. Since a letter-digit distinction is a highly overlearned concept, and since it requires analysis of only a single character, it may become available to the response process more quickly than a semantic categori- zation based on word meaning. Further speculation on this issue must await additional research on the boundary conditions under which the repeated stimulus inferiority effect can be reliably demonstrated.

In summary, Experiment 2 provides a useful confirmation of the relative time courses of several distinct response and semantic priming effects, by demonstrating these effects using very different stimulus materials from those of Experiment 1 and other letter-digit classification experiments. The findings of major importance to our understanding of attention are: (1) Response competition is an "early SOA" effect that is rapidly attenuated by target delay. (2) Facilitation by response-compatible flankers requires advance processing of the flankers, and reaches maximum influence at SOAs at which response-competition effects have largely disappeared. (3) When a semantic category distinction among target items varies within a response mapping, semantic congruity of flanker and target appears to be a prerequisite for facilitative priming, or perhaps semantic incongruity leads to an inhibition ef- 

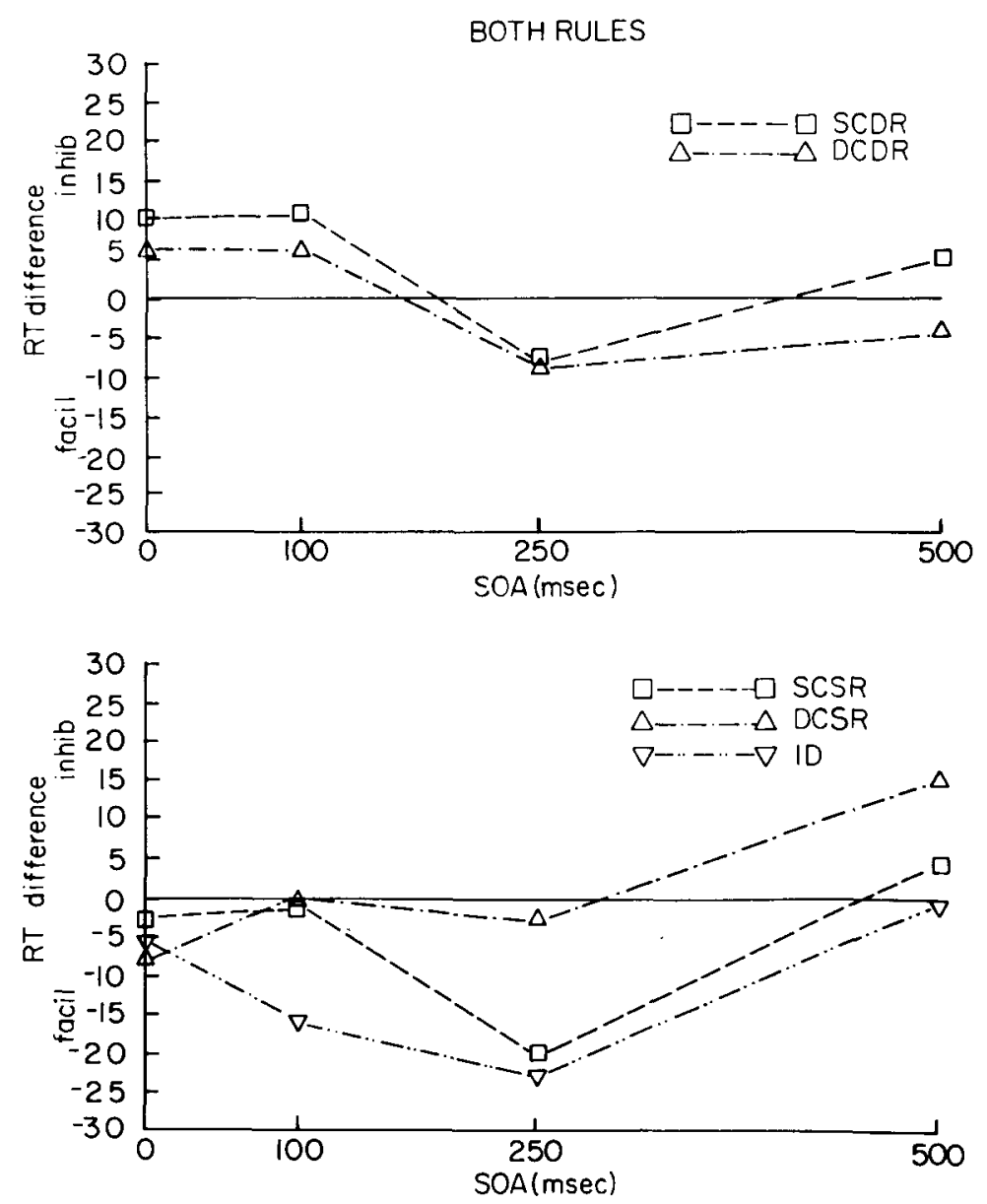

Figure 8. Differences in reaction time between the neutral and each of the other flanker conditions in Experiment 2, as a function of SOA. Differences are averaged across both classification rules.

fect offsetting potential facilitation from priming a compatible response.

\section{GENERAL DISCUSSION}

The present experiments add to a growing body of literature which suggests that perceptual priming effects of visual noise may reflect a variety of underlying processes with different temporal properties. These include effects related to the response mappings imposed by the specific experimental task, as well as categorization effects that stem from prior learning. It is important to recognize, however, that demonstration of these different effects over a range of stimulus materials is not sufficient to point to a single process model of attention or categorization, since there are a variety of plausible interpretations for some of these effects. It is, however, worthwhile to consider a few of these "plausible" interpretations, since they raise important issues for subsequent research.

\section{Response-Compatibility Effects}

The present results confirm a basic data pattern found in previous research. Both inhibitory effects of response competition and facilitation by compatible noise may be produced, but the former is most dominant with simultaneous noise and targets (but quickly diminishes with positive SOA), whereas the latter seems to require preexposure to the noise. This pattern seems sufficiently robust across different studies, despite the legitimate concern that a neutral stimulus may not possess constant neutrality across SOA levels (Jonides \& Mack, 1984), for the temporal asymmetry to seem to be an established general finding. It may be useful to relate the differing time courses of inhibition and facilitation to assumptions about attention derived from other recent experimental research.

First, it seems apparent that the varying of SOA may do more than simply change the relative times for processing flankers and targets. Temporal separation that pro- 
vides unique onset transients for the flankers could make them more fully attended and deeply processed than if they occurred in a simultaneous array with the target (Yantis \& Jonides, 1984). Second, there is a growing body of evidence that subjects use active inhibition processes to reduce deleterious effects of response competition. At least two different types of studies provide evidence for this: (1) studies by Grice and his colleagues (see Grice et al., 1984) that interpret differences in the shape of cumulative reaction time distributions as evidence for an ability to rapidly activate inhibition of response competitive processes, and (2) several studies (Neill \& Westberry, 1987; Tipper, 1985) that have shown that a specific stimulus that appears as a response-incompatible distractor on Trial $N$ is more slowly responded to as a target on Trial $N+1$, than it would be had the previous trial contained a different distractor.

Since it may allow both a head start in processing and greater assurance of more detailed processing, advance presentation of flankers could provide opportunities to more efficiently activate inhibition processes when conflict is detected. At the same time, the prior presentation of a compatible flanker, by both increasing the probability of its being attended and providing greater time for encoding, would enhance opportunities to benefit from priming.

It should be pointed out, however, that even though there is evidence that human observers can rapidly engage inhibition to suppress response competition, it is not necessary to postulate such dynamic attention changes simply to provide a plausible interpretation for why inhibition and facilitation from flankers have different time courses. Such an asymmetry could result simply from the dynamics of the response execution system itself, in that it may be more sensitive to disruption from activation by alternative response priming early in its activation, while being more resistant to being "pushed faster" by additional supportive information. Should these dynamics be reversed later in the time course of response activation, the response competition-facilitation asymmetry would result. Until more is learned about the microstructure of response activation, such a more parsimonious account of the facilitation-inhibition asymmetry cannot be discounted. ${ }^{5}$

\section{Category Congruity Effects}

Perhaps the most notable finding in this study is the demonstration of category-congruity effects that interact with response-compatibility and SOA. In both experiments, as well as in Experiment 3 of Flowers and Wilcox (1982), categorically incongruent but responsecompatible combinations produced considerably slower RTs than did either physically identical or categorically congruent combinations-but only at relatively long SOA levels. The interaction between response compatibility, category congruity, and SOA was not dependent on statistical comparisons involving a neutral baseline task, so it cannot be compromised by possible nonequivalence of the baseline condition across the longer SOA values. The finding that categorical distinctions within response groupings can affect priming thus appears to be a solid effect, and one which seems to depend largely on preexposure of the flankers.

Again, the fact that asychronous onset of the flankers relative to the target can allow for both increased likelihood of attention and detailed processing, plus a "head start" on whatever analysis takes place, seems to offer joint explanations for the dependence of the category congruity effect on SOA. With simultaneous onset of flankers and targets, there may be less likelihood of processing the flankers to the extent that category information is noted; and to the extent that such information may become available, it may arrive too late in the response activation process to have any impact on RT.

Evidence that categorical congruity can modify response competition effects is quite inconsistent among the tasks chosen for this study. Two of the letter-digit tasks seem to illustrate a category effect for the response-incompatible trials at 0 SOA, but the remaining tasks do not. Since response competition is a highly transitory effect, the occurrence of category effects may depend on whether categorical information can be sufficiently rapidly extracted from the flankers before the response competition is attenuated, a situation that could depend highly on subtleties of the nature of the categorical distinction, degree of learning, and so forth. With response-compatible trials, for which facilitative priming effects unfold over a longer time course, additional information about categorical structure may have a greater opportunity for impact on response speed.

Future research is needed to determine the extent to which active changes in the attentional state of the observer as opposed to asymmetric dynamics of response processes themselves account for differing time courses of the various priming effects described in this and related studies. Evidence for attentional state changes can perhaps be approached through techniques in which responses to a subsequent display sequence are evaluated as a function of what the subject had to filter (or perhaps benefit from) when responding to a previous display (Neill \& Westberry, 1987). For example, a potentially useful variant of such experiments involves the presentation of a probe detection task, immediately following a flanker task trial, to see whether sensitivity for detecting or discriminating a visual target in the spatial region previously occupied by a flanker is affected by flanker compatibility. The study of response-process dynamics may prove less direct and more difficult, although converging experiments on distributional properties of RTs (Grice et al., 1984), psychophysiological correlates (Coles, Gratton, Bashore, C. W. Eriksen, \& Donchin, 1985), and the microstructure of response execution (St. James, in press) may collectively prove useful. 


\section{REFERENCES}

Coles, M. G. H., Gratton, G., Bashore, T. R., Eruksen, C. W., \& Donchin, E. (1985). A psychophysical investigation of the continuous flow model of human information processing. Journal of Experimental Psychology: Human Perception \& Performance, 11, 529-553.

ERIKSEN, B. A., \& ERIKSEN, C. W. (1974). Effects of noise letters upon the identification of a target letter in a nonsearch task. Perception \& Psychophysics, 16, 143-149.

Erissen, C. W., \& Hoffman, J. E. (1973). The extent of processing of noise elements during selective encoding from visual displays. Perception \& Psychophysics, 14, 155-160.

ERIKSEN, C. W., ShULTZ, D. W. (1979). Information processing in visual search: A continuous flow conception and experimental results. Perception \& Psychophysics, 25, 249-263.

FLoWERS, J. H. (1980). Response priming effects in a digit naming task as a function of target-noise separation. Bulletin of the Psychonomic Society, 16, 443-446.

Flowers, J. H., Nelson, S., Carson, D., \& Larsen, L. (1984). Automatic and expectancy-based priming effects in a digit naming task. Joumal of Experimental Psychology: Human Perception \& Performance, 10, 65-74.

FLOWERS, J. H., \& Wilcox, N. (1982). The effect of flanking context on visual classification: The joint contribution of interactions at different processing levels. Perception \& Psychophysics, 32, 581-591.

Glaser, M. O., \& Glaser, W. R. (1982). Time course analysis of the Stroop phenomenon. Journal of Experimental Psychology: Human Perception \& Performance, 8, 875-894.

Glaser, W. R., \& Dungelhoff, F. (1984). The time course of pictureword inteference. Joumal of Experimental Psychology: Human Perception \& Performance, 10, 640-654.

Grice, G. R., Boroughs, J. M., \& Canham, L. (1984). Temporal dynamics of associative interference and facilitation produced by visual context. Perception \& Psychophysics, 36, 499-507.

GRICE, G. R., \& GWYNNE, J. W. (1985). Temporal characteristics of noise conditions producing facilitation and interference. Perception \& Psychophysics, 37, 495-501.

Hock, H., \& Petrasek, J. (1973). Verbal interference with perceptual classification: The effect of semantic structure. Perception \& Psychophysics, 13, 116-120.

LABERGE, D. (1981). Unitization and automaticity in perception. In J. H. Flowers (Ed.), Nebraska Symposium on Motivation: Cognitive processes (Vol. 28). Lincoln: University of Nebraska Press.

LABerge, D., \& SAMUels, S. J. (1974). Toward a theory of automatic information processing in reading. Cognitive Psychology, 6, 293-323.

NEELY, J. H. (1977). Semantic priming and retrieval from lexical memory: Roles of inhibitionless spreading activation and limited capacity attention. Journal of Experimental Psychology: General, 106, 226-254.

NeILL, W. T., \& WestberRY, R. L. (1987). Selective attention and the suppression of cognitive noise. Journal of Experimental Psychology: Learning, Memory, \& Cognition, 13, 327-334.

PosNer, M. I., \& SNYDER, C. R. R. (1975). Attention and cognitive control. In R. E. Solso (Ed.), Information processing and cognition: The Loyola Symposium (pp. 55-85). Hillsdale, NJ: Erlbaum.

Proctor, R. W., \& Forer, G. W. (1985). Repeated-stimulus superi- ority and inferiority effects in the identification of letters and digits. Perception \& Psychophysics, 38, 125-134.

ST. JAmES, J. D. (in press). Observations on the microstructure of response competition. Perception \& Psychophysics.

Shaffer, W. O., \& LABERGE, D. (1979). Automatic and semantic processing of unattended words. Journal of Verbal Learning \& Verbal Behavior, 18, 413-426.

STANOVICH, K. E., \& WEST, R. F. (1983). On priming by a sentence context. Journal of Experimental Psychology: General, 112, 1-40.

TAYLOR, D. A. (1977). Time course of context effects. Journal of Experimental Psychology: General, 106, 404-426.

TIPPER, S. P. (1985). The negative priming effect: Inhibitory priming by ignored objects. Quarterly Journal of Experimental Psychology, 37A, 571-590.

YANTIS, S., \& Jonides, J. (1984). Abrupt visual onsets and selective attention: Evidence from visual search. Journal of Experimental Psychology: Human Perception \& Performance, 10, 601-621.

\section{NOTES}

1. Unlike a letter-digit categorical distinction, locations within the alphabet list and within the digit list are not clearly equivalent psychological dimensions when compared with each other. Thus for Rule 3, the category congruity distinctions between SCSR and DCSR are likely to be more salient than the distinctions between SCDR and DCDR.

2. Effects of flanker type, SOA, and their interaction were statistically significant for all three classification nules $(p<.01$ or less, except for flanker type for Classification Rule 2, which was significant at $p<.025$ )

3. Consistent with the overall ANOVA, significant interactions between response compatibility and SOA $[F(4,108)=36.37, p<.001]$ and between semantic congruity and SOA $[F(4,108)=2.47, p<.05]$ were noted, which I assume to be largely attributable to the attenuation of all flanker effects at the longest SOA.

4. Admittedly, it is an overstatement to say that the categorical distinctions vary in a truly orthogonal manner with response compatibility for these stimulus sets, since silver and gold are precious metals while iron and brass are not; maple and birch are hardwoods, spruce and pine are not, and so forth. Thus, one should not assume that the same category versus different category distinctions are completely psychologically equivalent on response-compatible and response-incompatible trials, just as such an assumption was not justified for Rule 3 of the previous experiment. This nonequivalence could reasonably be expected to diminish category effects on response-incompatible trials. It has been my experience in several previous experiments that subjects faced with a complex sorting task that involves familiar stimuli will impose their own conceptual structures upon the set if an obvious grouping structure is not present. To minimize variability of idiosyncratic organizational schemes and to promote more rapid learning of the response assignments, these "secondary" classification attributes were purposely incorporated into the stimulus set.

5. I am indebted to comments from the editor, C. W. Eriksen, for helping me frame this issue.

(Manuscript received August 12, 1988; revision accepted for publication August 21, 1989.) 